\title{
Effect on Communication System of Dispersive Multiplexing in Optical Fiber
}

\author{
Zhengqin Dong ${ }^{1, *}$ and Yun $\mathrm{Lu}^{2}$ \\ ${ }^{1}$ School of Computer Science and Technology, Wuhan University of Technology, Wuhan, China, 430074 \\ ${ }^{2}$ School of Naval Architecture and Ocean Engineering, Huazhong University of Science and Technology, Wuhan, China, 430074 \\ * Corresponding author: 15926424120@163.com
}

\begin{abstract}
In order to research on the transmission effect on communication system of dispersive multiplexing in optical fiber, the concept is applied to data transmission over multimode optical fiber, and the result is an optical multiplexing that can increase the capacity of such fiber in the engineering. Experiment result shows that the proposed method can thus improve the overall system performance substantially.
\end{abstract}

\section{Introduction}

From the standpoint of information theory, multimode optical fiber (MMF) has more information capacity than singlemode optical fiber (SMF). However, this assertion becomes meaningless when one tries to send a high data rate down any sizable length of multimode fiber, owing to the effects of modal dispersion, which severely limit the achievable data rates in current MMF transmission systems. The information theory viewpoint relies on the notion that the additional spatial modes in the transmission medium provide additional degrees of freedom and, therefore, additional capacity.

In practice, however, there is no simple way to independently address and detect the individual modes of the fiber (which number in the hundreds), and this, coupled with the presence of mode-mixing during propagation (often present to some degree), makes it nearly impossible to exploit the additional capacity in any straightforward manner. Because of their cost advantages, systems employing MMF are finding increased use in certain data networking applications, and MMF promises to replace copper as the transmission medium of choice for future high-speed local area networks, perhaps eventually reaching the desktop. Any practical technique that could effectively use the spatial degrees of freedom in the fiber to improve transmission bandwidth could potentially find extensive application in these future systems.

We describe a transmission scheme that uses the effects of modal dispersion to enhance the transmission capacity of MMF. The method does not require orthogonal coupling to the individual modes of the fiber and can tolerate some degree of mode-mixing. The technique is based on recently developed ideas in the field of wireless communications. Using information theory, Foschini and Gams have shown that a rich multipath scattering environment pro-vides a dramatic capacity enhancement for wireless systems employing multiple antennas at both the transmitter and receiver. Under conditions of sufficiently strong scattering, the information capacity of a multiple transmit receive system scales linearly with the number of transmitters, even as the total transmit power is held constant. This extraordinary result occurs, in a fundamental sense, because the system is able to use the spatial degrees of freedom inherent in the scattering environment to improve the capacity. Experimental work in this area has resulted in multiple antenna systems with spectral efficiencies as high as 40 bits, a value unobtainable by conventional transmission methods $(7,8)$. We demonstrate here that these basic concepts can be applied to optical transmission in multimode fiber, in a system using multiple lasers and multiple detectors. Although there are important differences between the optical and wireless versions of this technique, we show that for the optical system, the most important property, the linear scaling of the capacity with the number of transmitters, is preserved.

\section{The Model and Framework}

The transmission system (Fig. 1) consists of $\mathrm{N}$ lasers coupled together onto a single multimode fiber and received at the end of this fiber link by $\mathrm{M}$ detectors. $\mathrm{N}$ independent bit streams are first modulated onto identical radio frequency (RF) carriers, and these electrical signals modulate the $\mathrm{N}$ lasers. The RF modulation format is arbitrary, the data clocks in 
each arm are synchronized, and the symbol rate of the individual bit streams is constrained to operate within the bandwidth of the fiber. At the receiver, the optical power is split evenly between the $\mathrm{M}$ detectors, which convert the optical signals to electrical signals through direct detection. Each detector receives power from all $\mathrm{N}$ transmitting lasers. These signals are demodulated by coherent microwave detection, and the resulting $\mathrm{M}$ baseband signals (which contain a mix of all of the data) are sent to a signal processing circuit that recovers the original $\mathrm{N}$ data streams. No wavelength division multiplexing (WDM) is used in the combining or splitting of the optical signals, i.e., the wavelengths of the transmitting lasers do not have to be precisely specified.

The system works because of a process referred to here as "modal-coupling diversity." At the input to the system, each laser excites a slightly different modal power distribution within the fiber. There will be a tendency toward this type of diversity regardless of the actual technique used to couple the $\mathrm{N}$ beams together. At the output of the fiber, further modal-coupling diversity causes each detector to receive power from a slightly different distribution of modes (even when the coupling optics is designed to split the total optical power equally).

One can define an M X N channel matrix that characterizes the system: Each element of this matrix is a complex number that relates the (electrical) magnitude and phase of the received RF carrier in a given detector to that transmitted from a given laser. These matrix elements are determined by the impulse response of the channel. As a result of modal dispersion, a carrier injected into the front end of the system will appear as a series of randomly phased sine waves at the receiver. Under conditions where sufficient modal dispersion exists in the fiber (such that the impulse response length which is at least the RF cannier period), the elements of the transmission matrix can be modeled as uncorrelated Gaussian random variables. This is a consequence of the large number of modes, the dispersion properties of the fiber, and the modal coupling diversity and is analogous to the Rayleigh fading model used for multipath propagation in wireless systems. Measurements of actual MMF transmission have confirmed this property (Fig. 2). For a 2-km length of 62.5-pm fiber, independent Rayleigh fading statistics are realized for RF carriers greater than $-500 \mathrm{MHz}$. It is this similarity between the optical and wireless regimes that suggests the applicability of multiple antenna theories to MMF transmission.

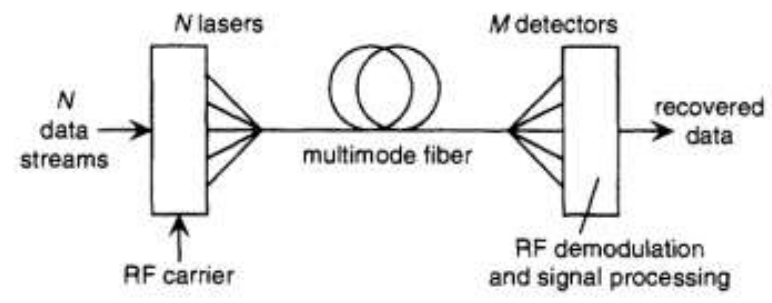

Fig. 1. Diagram of the proposed optical transmission system.

The transmitter and receiver "diversity" is essential for the system shown in Fig. 1 to work. In order to recover the transmitted information, the channel matrix must be known (channel estimation can be accomplished with a series of training bits preceding the data stream) and it must be invertible. A single-mode fiber, for example, will necessarily produce a noninvertible channel matrix. For a MMF, if the elements of the matrix are partially correlated (because of insufficient diversity or insufficient modal dispersion), the matrix will be poorly conditioned, leading to difficulties in achieving error-free data recovery. These issues can be quantified by using information theory to derive the capacity of the transmission system. The formalism used to derive capacity for multiple-antenna wireless systems can be applied here, but only after a modification of the basic assumptions underlying the details of the transmission. The most important difference between the optical and wireless regimes occurs at the receiver. In the wireless system, the total received power increases as the number of receiving antennas increases. In the optical system, a fixed amount of optical power anises at the end of the fiber, and this power is split between the $M$ detectors, reducing the average received signal-to-noise ratio (SNR). The power splitting at the receiver can be compensated at the transmitter, with the perchannel SNR preserved by setting N = IM (an equal number of lasers and detectors). This assumes lossless coupling of the $\mathrm{N}$ lasers to the fiber, a requirement that is physically allowed for multimode fiber. This requirement is in contrast to the wireless system, where the total transmitted power is fixed independent of $\mathrm{N}$. 


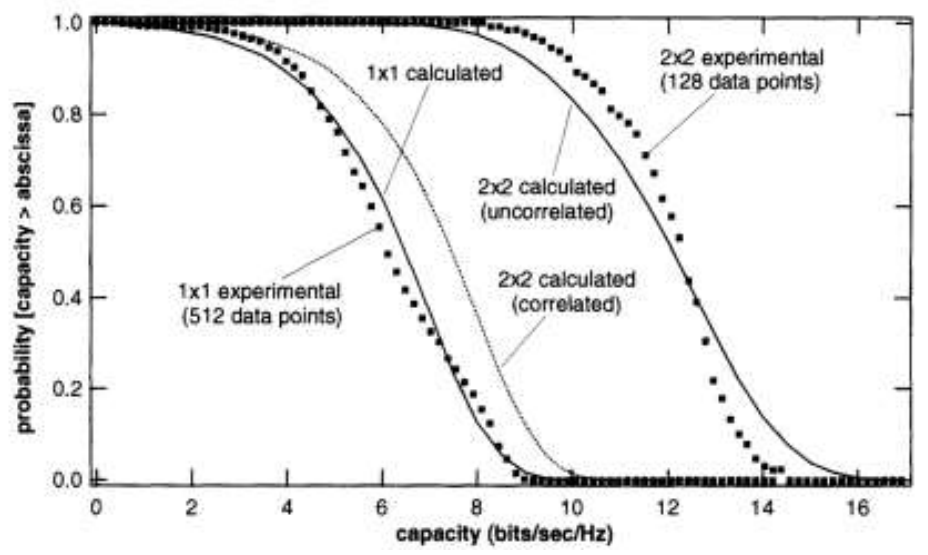

Fig. 2. Squares Cumulative distribution functions (CDFs) of capacity.

\section{Experiment Results and Discussion}

The linear scaling of capacity with $\mathrm{N}$ is significant, as it implies that one can multiplex independent signals onto "virtual" spatial channels that are resolvable through the dispersive properties of the modes. The depraving of the RF carrier at high frequencies enables this to happen; however, this depraving also introduces a notable power penalty relative to baseband transmission to $20 \mathrm{~dB}$ for $1-\mathrm{km}$ MMF transmission). This must be overcome to realize true capacity enhancement in the link. Because capacity degrades logarithmically (not linearly) with lost power (- 1 bit s-' Hzl for each $3 \mathrm{~dB}$ of power), this is achieved with a relatively modest number of transmitters. For $\mathrm{p}=21$ and $9 \mathrm{~dB}$, capacity enhancements are realized for $\mathrm{N}>3$ and $\mathrm{N}>5$, respectively, assuming an 18-dB power penalty. We have built a 2 X 2 experimental demonstration of this technique, operating at a relatively low bit rate (50 Mbits per channel), but clearly demonstrating the feasibility of this type of multiplexing. For simplicity, a conventional $1 \mathrm{X} 2$ splitter is used to couple the two lasers into the fiber, introducing $3 \mathrm{~dB}$ of optical power loss which, though tolerable in this initial experiment, will need to be eliminated for higher bit-rate systems. Two independent data streams are binary phase shift key (BPSK) modulated onto identical RF carriers $(-1 \mathrm{GHz})$, and these signals are used to directly modulate the drive current of the lasers. At the receiver, the detectors convert the received optical signals to RF electrical signals and these are quadrature phase shift key (QPSK) demodulated to recover the baseband signals. QPSK demodulation is required to recover the complete phase information of the received signal. The recovered baseband data are fed to an analog processing circuit, described below, which recovers the transmitted bit streams.

The constellation diagrams display the phase space of the received data and serve to illustrate the basic properties of the transmission. For back-to-back transmission (top plots), there is negligible modal dispersion, and we observe a three-point constellation in detector 1 . The outer two points correspond to the data pairs $(1,1)$ and $(-1,-1)$, while the center point consists of two overlapping data pairs, $\{(I,-1),(-1,1)\}$. This is indicative of two interfering (and unresolvable) BPSK signals and results from an attempt to send two independent signals over a single channel. Although we do observe a slight splitting of the center point in detector 2 (attributable to residual "power-splitting" diversity in the MMF couplers), the channel matrix for the top plots is poorly conditioned (condition number $>20$ ), and data recovery would require a very high SNR. In the bottom plots, $1 \mathrm{~km}$ of MMF has been inserted into the system. The received constellations now consist of four clearly resolved points in each detector, and the constellation seen by each is different, demonstrating the diversity present in the system.

The condition number of the channel matrix in this example is 4.5. Application of the signal-processing algorithms results in recovery of the transmitted data (Fig. 3B). The signal-processing steps required for data recovery consist of estimating the channel matrix and determining, then applying, a set of complex weights to cancel the interfering signals. The optical channel was observed to be relatively stable, suggesting that the channel can be estimated, inverted, and tracked "off-line" (at rates much slower than the bit rate). Even so, the degree of signal processing required to apply the complex weights at gigabit per second data rates is daunting and led us to consider an analog (rather than digital) approach. We have constructed a real-time analog processing circuit, using 500-MHz bandwidth analog multipliers.

The processor recovers a single dimension in phase space (sufficient for BPSK) for one channel. The processor bandwidth in the initial implementation was limited to $50 \mathrm{MHz}$ to simplify circuit layout. Channel estimation is accomplished by viewing the eye diagram in real-time on an oscilloscope and adjusting the weights (four potentiometers on the circuit board) until the interfering channel is eliminated. In the experiments, each of the transmitted channels is recovered in sequence (two of these circuits would be required in a complete system). These curves show a small $(-1.2 \mathrm{~dB})$ optical power penalty associated with the presence of the interfering channel. Although not shown on the plot, BERs below $10^{-9}$ have been measured. 


\section{References}

1. Wei Dai, Mostafa Bassiouni. An improved task assignment scheme for Hadoop running in the clouds. Journal of Cloud Computing, (2013), pp. 21-33.

2. Wei Kuang Lai, Yi-Uan Chen, Tin-Yu Wu, Mohammad S. Obaidat. Towards a framework for large-scale multimedia data storage and processing on Hadoop platform. The Journal of Supercomputing, (2014), pp. 681-696.

3. Zhijian Chen, Wenhai Luo, Dan Wu, Xiang Huang, Jian He, Yuanhuan Zheng, Di Wu. Exploiting application-level similarity to improve SSD cache performance in Hadoop. The Journal of Supercomputing, (2014), pp. 703-715.

4. Benrun Huang, Li Xia, Zhengguo Wu, Weiping Zhou. Control Strategy of A Dynamic Voltage Restorer (DVR) Based on Line Voltage Compensation. Energy Procedia, (2011), pp. 13-26.

5. Wei Dai, Mostafa Bassiouni. An improved task assignment scheme for Hadoop running in the clouds. Journal of Cloud Computing, (2013), pp. 21-33.

6. Wei Kuang Lai, Yi-Uan Chen, Tin-Yu Wu, Mohammad S. Obaidat. Towards a framework for large-scale multimedia data storage and processing on Hadoop platform. The Journal of Supercomputing, (2014), pp. 681-696. 